\title{
Dechlorination of 2,4-dichlorophenol by nickel nanoparticles under the acidic conditions
}

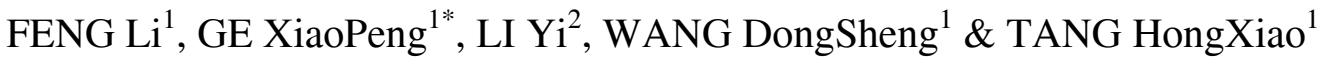 \\ ${ }^{1}$ State Key Laboratory of Environmental Aquatic Chemistry, RCEES, Chinese Academy of Sciences, Beijing 100085, China; \\ ${ }^{2}$ Nanotechnology Center, Central Iron and Steel Research Institute, Beijing 100081, China
}

Received February 14, 2011; accepted May 4, 2011

\begin{abstract}
Metal nanoparticles are effective for remediation of contamination with a range of compounds including chlorinated organics. However, the sorption process of the passivation oxide layers on the metal nanoparticle surfaces may result in incomplete degradation of contaminants. This phenomenon can be prevented by an acidic washing procedure or reaction in an acidic medium. In this paper, nickel nanoparticles manufactured via the carbonyl powder process were analyzed using scanning electron microscopy, transmission electron microscopy, X-ray diffraction and energy-dispersive X-ray spectroscopy. The sorption and degradation of 2,4-dichlorophenol (2,4-DCP) by nickel nanoparticles under acidic conditions was then investigated. Transmission electron microscopy and XRD results showed that the nickel nanoparticles range in size from 10 to $20 \mathrm{~nm}$, and a thin passivation layer of $\mathrm{NiO}$ is present on the surface. This oxide layer can be removed by pretreatment washing with acidic solutions. It was indicated that dechlorination was the key reaction pathway for degradation of 2,4-DCP by nickel nanoparticles under acidic conditions. The main degradation products were 4-Chlorophenol, 2-Chlorophenol, and Phenol, and among these, Phenol was dominant. The acidic medium promoted degradation by providing an appropriate $\mathrm{pH}$, and $\mathrm{H}^{+}$may be involved in the reaction. Dechlorination of 2,4-DCP by nickel nanoparticles under the acidic condition follows the second order kinetic model, and the rate constants at 298, $306,316 \mathrm{~K}$ are $0.02,0.2$ and $0.3(\mathrm{~g} \mathrm{Lh})^{-1}$, respectively.
\end{abstract}

nickel, nanoparticles, 2,4-dichlorophenol, dechlorination, sorption, acid conditions

Citation: Feng L, Ge X P, Li Y, et al. Dechlorination of 2,4-dichlorophenol by nickel nanoparticles under the acidic conditions. Chinese Sci Bull, 2011, 56: 2258-2266, doi: 10.1007/s11434-011-4549-1

Metal nanoparticles, especially those of iron, offer many advantages for remediation of contamination with chlorinated organic pollutants because of their small particle size, large specific surface area [1,2], excellent adsorption and high reduction reactivity [3]. In recent years, nanoscale zero-valent iron has been used for the treatment of environmental pollution [4,5]. It is a very effective reducing agent for dehalogenation and can catalytically reduce various halogenated organics into non- or low-toxic compounds, including the persistent organic pollutants chlorohydrocarbons [6,7], chloroaromatics [8], chlorophenol [9], organochloride pesticides (OCPs) [10] and polybrominated diphenyl ethers (PBDEs) [11].

*Corresponding author (email: xpge@rcees.ac.cn)
The industrial carbonyl powder process is a metal powder refining technology with high selectivity that produces metal powder by pyrolysis of organometalic carbonyl compounds. This method is mainly used in iron and nickel metal powder preparation, and produces high purity metal powders that could be used in environmental remediation. Hou Chunfeng et al. [12]. investigated the degradation of 2,4dichlorophenol $(2,4-\mathrm{DCP})$ by nanoscale iron ultrafine carbonyl powders, and found that without activation by washing with acidic solutions, 2,4-DCP was mainly removed by adsorptive process of oxide passivation layers on the particle surfaces. Also, no degradation products were detected by high-performance liquid chromatography (HPLC). However, the high adsorption capability of metal nanoparticles has both advantages and disadvantages. While adsorption fa- 
cilitates dehalogenation, it is also the main contributor to incomplete degradation of halocarbons. The persistent organic pollutants adsorbed on nanoscale metal particle surfaces may be present for a very long time in the environment and be transferred long distances with the metal nanoparticles in water bodies, leading to the mass diffusion of pollutants. Previous research showed that sorption was mainly because of oxide layer formation during the reaction, and acidic conditions could limit the formation of oxide layers [13].

Because iron nanoparticles can be oxidized when exposed to air and spontaneous combustion may occur during storage [14], controlled oxidation of iron is usually performed in the manufacturing process to produce a passivation layer and allow the collection of ultrafine carbonyl powders as the final product. This leads to large mass loss in the acid-washing procedure during application of the nanoparticles. By comparison, even with only very thin oxide layers on their surfaces, nickel nanoparticles are less reactive than those of nanoscale iron. However, they still share similar physicochemical properties. In this study, nickel nanoparticles manufactured via the carbonyl powder process were characterized by scanning electron microscopy (SEM), transmission electron microscopy (TEM), X-ray diffraction (XRD) and energy-dispersive X-ray spectroscopy (EDS). The sorption and degradation of 2,4-DCP by nanoscale nickel under acidic conditions was studied to determine the dechlorination mechanism and explore the potential of nanoscale nickel for environmental remediation.

\section{Materials and methods}

(i) Reagents and materials. 2,4-DCP (Sinopharm Chemical Reagent Corporation, Shanghai, China), phenol standard solution (National Institute of Metrology, Beijing, China), methanol (Fisher Scientific, Fair Lawn NJ), and purified water (Wahaha Group, Tianjin, China) were obtained for this study. A combined $0.45 \mu \mathrm{m}$ fibrous microfiltration membrane was purchased from Beijing Shenghe Chengxin Membrane Science and Technology Development Center (Beijing, China) and used for all solid/liquid phase separations. Nickel nanoparticles manufactured by thermolysis and vapor deposition of nickel carbonyl under $\mathrm{N}_{2}$ were obtained from the Central Iron and Steel Research Institute (Beijing, China). All reagents used in this study were analytical grade.

(ii) Characterization of nickel nanoparticles. The nickel nanoparticles were characterized by powder XRD on a D/ Max-RC diffractometer (Rigaku, Tokyo, Japan) using $\mathrm{Cu}$ $\mathrm{K} \alpha$ radiation $(\lambda=1.5418 \AA)$. XRD data on the crystallinity of nickel nanoparticles were collected in scan mode with a step length of 0.033 and a scan angle of $5^{\circ}-100^{\circ}$. The accelerating voltage and the applied current were $40 \mathrm{kV}$ and $40 \mathrm{~mA}$, respectively. The specific surface area, aperture and volume were measured by the $\mathrm{N}_{2}$ /BET method (ASAP 2000, Micromeritics, Norcross, GA). Before analysis, samples were pretreated at $90^{\circ} \mathrm{C}$ for $1 \mathrm{~h}$ and at $350^{\circ} \mathrm{C}$ for $3 \mathrm{~h}$. Morphology and shape of the nickel nanoparticles were observed by TEM. Before TEM observation, the nickel nanoparticles were dispersed in ethanol solution by ultrasonic agitation. The suspensions were dropped onto copper grids coated with a perforated carbon film. The grids were dried in air at room temperature and examined on S-570 TEM (Hitachi, Tokyo, Japan). The apparent particle size distribution in aqueous suspension was determined by a particle size analyzer (Mastersizer 2000, Malvern, Worcestershire, UK). The elemental composition of the surface of the nickel nanoparticles was analyzed by EDS using S-3000N SEM (Hitachi, Tokyo, Japan) equipped with an energy dispersive $\mathrm{X}$-ray detector (USA).

(iii) Degradation of 2,4-DCP by nickel nanoparticles without acid-wash pretreatment. Batch experiments were carried out in $200 \mathrm{~mL}$ conical flasks sealed with parafilm. The nickel nanoparticles (15 g/L) without acidic washing were ultrasonically dispersed for $20 \mathrm{~min}$ in water and used for 2,4-DCP degradation experiments. $5 \mathrm{mg} / \mathrm{L} 2,4-\mathrm{DCP}$ of concentrations were applied in the experiments. Three parallel samples were taken and control samples were prepared without adding the nickel nanoparticle powders. All flasks were placed on a thermostatic orbital shaker (HZQ-C, Harbin, China) at $33 \pm 2^{\circ} \mathrm{C}$ and shaken at $200 \mathrm{r} / \mathrm{min}$ for a set time. At each sampling time, an aliquot was taken from each flask and solid/liquid phase separation was performed. The supernatant solutions were analyzed for 2,4-DCP and its dechlorination products.

(iv) Degradation of 2,4-DCP by nickel nanoparticles under neutral and acidic conditions after acid-wash pretreatment. An oxide layer is usually present on nickel nanoparticle surfaces because of the manufacturing process. A passivation step is usually included to collect the ultrafine carbonyl powders as the final product. Therefore, acid-wash pretreatment is required to remove the oxide passivation layers when nickel nanoparticles are applied. According to initial experiments, treatment with dilute sulfuric acid $\left(\mathrm{H}_{2} \mathrm{SO}_{4}\right)$ may result in loss of approximately $15 \%$ of the nickel, and this was taken into account in the calculation of its dose for the experiments. Nickel nanoparticle suspensions were pretreated as follows: appropriate amounts of nickel nanoparticles were weighed and transferred to conical flasks, $\mathrm{H}_{2} \mathrm{SO}_{4}$ : water $(1: 200, v / v)$ was added to the flasks, the flasks were stirred on a magnetic stirrer (90-4, Shanghai Zhenrong Scientific Instruments Co., Shanghai, China) for $1 \mathrm{~h}$, and the nickel particles were washed with purified water several times to remove any residual acidity after the acid treatment. The samples were then diluted with purified water to obtain nickel nanoparticle suspensions at the required concentrations.

Samples for $5 \mathrm{mg} / \mathrm{L}$ 2,4-DCP degradation experiments were then prepared and analyzed as in Section (iii), but the 
samples were shaken at three different temperatures $(25 \pm$ $2^{\circ} \mathrm{C}, 33 \pm 2^{\circ} \mathrm{C}$, and $43 \pm 2^{\circ} \mathrm{C}$ ), respectively.

After the acid-wash experiments, the nickel nanoparticles were washed with ultrapure water to be used in an experiment under neutral conditions. As for the experiment under acidic conditions, solution of $\mathrm{H}_{2} \mathrm{SO}_{4}$ : water $(1: 200, v / v)$ was added dropwise to maintain the $\mathrm{pH}$ at 3-4.

Chlorinated phenols in the samples were quantified by using Waters 1525 Binary Pump HPLC equipped with a 2487 detector and C18 column (Waters Co., Milford, MA). A mixture of methanol and water $(70: 30, v / v)$ was used as the mobile phase at a flow rate of $1.0 \mathrm{~mL} / \mathrm{min}$. The sample loop was $20 \mu \mathrm{L}$. The compounds were detected using a UV detector at $280 \mathrm{~nm}$.

(v) Desorption of 2,4-DCP and phenol from nickel nanoparticles. To examine the sorption characteristics of 2,4-DCP and its dechlorinated products by nickel nanoparticles, desorption experiments were performed with the 2,4-DCP or phenol loaded nickel nanoparticles. After the loading experiment by 2,4-DCP or phenol the nickel nanoparticle suspensions were separated by centrifugation. Ultrapure water $(200 \mathrm{~mL})$ was added to the solid phase and mixed for desorption experiments under the same conditions. At each sampling time, an aliquot was taken from each flask and solid/liquid phase separation was performed. The supernatants were analyzed for 2,4-DCP or phenol concentrations.

\section{Results and discussion}

\subsection{Characterization of the nickel nanoparticles}

Morphology, particle size and composition are important in determining the chemical and physical properties of nanoparticles. These fundamental characteristics of the nickel nanoparticles were investigated using the BET specific surface area, TEM, XRD and EDS analysis.

(i) Morphology and BET specific surface area. SEM and TEM images of the nickel nanoparticles are shown in Figure 1. The SEM image was obtained directly from a sample of nickel ultrafine powders without dispersion in any solvent, while the TEM observation was of nickel
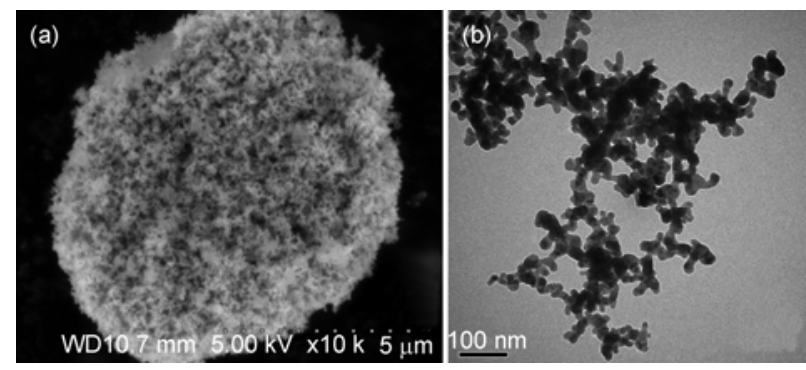

Figure 1 SEM (a) and TEM (b) micrographs of the nickel nanoparticles. nanoparticles dispersed in ethanol. The SEM micrograph with low magnification showed that the nickel nanoparticles formed large ball-like clusters, and chain-like aggregates of individual spherical particles are obvious from the close-up TEM image. The individual spherical particles were on average $10-20 \mathrm{~nm}$ in diameter. The BET specific surface area was $32.40 \mathrm{~m}^{2} / \mathrm{g}$, which is nearly four times larger than that for the iron particles made from the same carbonyl powder process $\left(7.075 \mathrm{~m}^{2} / \mathrm{g}\right)$ and 30 times larger than normal microscale iron particles $\left(0.9 \mathrm{~m}^{2} / \mathrm{g}\right.$ [5]).

(ii) Composition and structures. The structure of nickel nanoparticles and their phase composition were investigated by XRD and EDS, respectively. Figure 2 shows the XRD patterns of nickel nanoparticles. Four diffraction peaks $(2 \theta)$ were identified at $44.498^{\circ}, 51.877^{\circ}, 76.457^{\circ}$ and $93.106^{\circ}$, respectively. Bragg's law is expressed as $n \lambda=2 d \sin \theta$, where $n$ is an integer and in this case $n=1, \lambda$ is the wavelength of the incident radiation, $d$ is the spacing between the planes in the atomic lattice, and $\theta$ is the angle between the incident radiation and the scattering planes. Using Bragg's law the $d$ spacings between the crystal faces were calculated at 2.034, $1.760,1.245$ and $1.061 \AA$ for the $44.498^{\circ}, 51.877^{\circ}, 76.457^{\circ}$ and $93.106^{\circ}$ peaks, respectively. Referring to XRD patterns of standards these peaks were assigned to the diffraction 111 (2.035 ̊), 200 (1.762 ̊), 220 (1.246 ̊) and 311 (1.063 $\AA$ ) crystal faces of the face-centered cubic lattice of nickel.

The two minor peaks $(2 \theta)$ at $37.282^{\circ}$ and $63.563^{\circ}$ were attributed to the diffraction of $\mathrm{NiO}$; however, these diffraction peaks were extremely weak, which indicates that the nickel nanoparticles used in this investigation had a very low content of $\mathrm{NiO}$. This also implies that a very thin oxide passivation layer is present on the nickel surface.

The Scherrer formula is expressed as $\tau=K \lambda / \beta \cos \theta$, where $K$ is the shape factor, $\lambda$ is the $\mathrm{X}$-ray wavelength $(0.15405 \mathrm{~nm}), \beta$ is the line broadening in radians at half the maximum intensity, $\theta$ is the Bragg angle, and $\tau$ is the mean size of the ordered (crystalline) domains. The shape factor is a somewhat arbitrary value that falls between 0.87-1 depending on the particle grain size, and is used to correlate

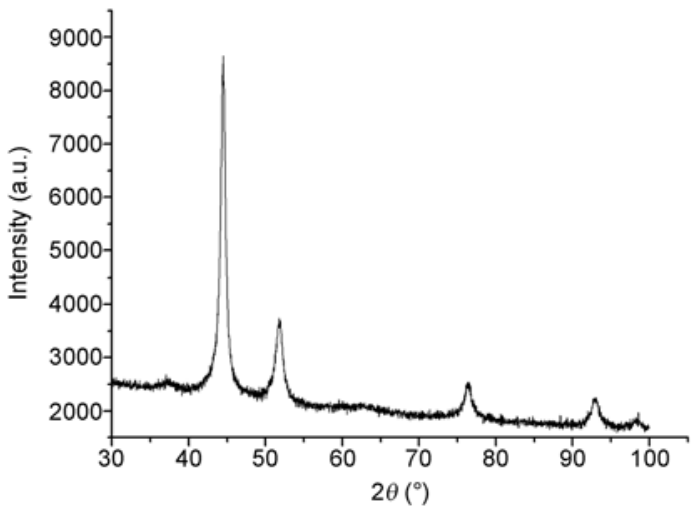

Figure 2 XRD patterns of the nickel nanoparticles. 
the size of sub-micrometer particles, or crystallites, in a solid to the broadening of a peak in the diffraction pattern. The grain size of nickel nanoparticles was calculated to be $11.3085 \mathrm{~nm}$ using the Scherrer formula, which agrees with the 10-20 nm diameter obtained by TEM.

EDS analysis was performed on the nickel nanoparticles to confirm their composition. A large nickel signal was detected with minor contributions from $\mathrm{O}$ and $\mathrm{C}$ (Table 1). The mass ratio of $\mathrm{C}: \mathrm{O}: \mathrm{Ni}$ was $3.11: 4.40: 92.49$, and the corresponding atomic ratio was $12.28: 13.04: 74.68$, indicating that a $\mathrm{NiO}$ layer and carbon atoms were present in the sample, which are associated with the manufacturing process of nickel nanoparticles.

\subsection{Comparison of 2,4-DCP degradation by nickel nanoparticles under different conditions}

The effects of oxide layers and solution $\mathrm{pH}$ on the dechlorination of 2,4-DCP were investigated with a series of experiments. Three systems with different media and reaction conditions were used. In the first, ultrafine nickel powders without acid-wash pretreatment were used directly in the dechlorination of 2,4-DCP after dispersion in solution, while in the other two cases the nickel nanoparticles were activated by washing with acidic solutions. The so-called neutral conditions were applied after the activated nanoscale nickel suspension was subsequently washed with ultrapure water to the neutral $\mathrm{pH}$ value. The third system was obtained by adding dilute sulfuric acid to the activated nickel nanoparticle suspension dropwise to adjust and maintain the $\mathrm{pH}$ at 3-4 (acidic conditions) in the dechlorination reaction.

The concentrations of 2,4-DCP and its removal rates with reaction time by nickel nanoparticle suspensions under the three different conditions are presented in Figure 3. Figure 3(a) and (b) shows the results for nickel nanoparticles without acid-wash pretreatment and Figure 3(c) and (d) is for the two suspension systems after acid-wash pretreatment. The 2,4-DCP concentration decreased with reaction time, especially for those in acidic conditions. In this case, the 2,4-DCP concentration was reduced to almost zero within a few hours. For the nickel nanoparticles without acid-wash pretreatment and for the neutral conditions, however, the concentration changed relatively slowly and some fluctuations occurred with the equilibration reached in about $20 \mathrm{~h}$. A $5 \mathrm{~h}$ period of hysteresis was evident for 2,4-DCP adsorption by nickel nanoparticles without acid-wash pretreatment

Table 1 EDS results for the nickel nanoparticles

\begin{tabular}{ccc}
\hline Elements & wt\% & at\% \\
\hline $\mathrm{C}$ & 3.11 & 12.28 \\
$\mathrm{O}$ & 4.40 & 13.04 \\
$\mathrm{Ni}$ & 92.49 & 74.68 \\
\hline
\end{tabular}

before significant removal began, which was probably because of the hydrophobic nature of nickel nanoparticle surfaces and the thin oxide layers thereon. Close-up figures in the initial $5 \mathrm{~h}$ period were given in the insets of Figure 3(a) and (b) to show more details. About $40 \%$ of 2,4-DCP removal was obtained in this case. By contrast, the neutral suspension system after acid washing gave up to $70 \%$ of $2,4-\mathrm{DCP}$ reduction that would decrease as the reaction continued. In both these cases, no 2,4-DCP degradation products were detected by HPLC over the entire experimental period. For the nickel nanoparticle suspension under acidic conditions ( $\mathrm{pH} 3-4)$, the 2,4-DCP concentration decreased rapidly and large quantities of degradation products of phenol etc. were detected. This indicates that acidic conditions can largely promote the dechlorination reaction and result in complete removal of 2,4-DCP.

To further evaluate the sorption of 2,4-DCP by nickel nanoparticle suspensions in the above three different systems, desorption experiments were performed on the loaded nickel nanoparticles after separation by centrifugation. The results are presented in Figure 4. As can be seen, 2,4-DCP was obviously released from the nickel nanoparticles for both the neutral suspension and the one without acid-wash pretreatment while for the loaded nickel nanoparticles under acidic conditions only trace amounts of 2,4-DCP were detected in the desorption solution. This is consistent with the adsorption process and confirms an incomplete degradation in the former two systems.

From the desorption experiments, characteristic features of 2,4-DCP desorption from nickel nanoparticle suspensions were obvious. In the first, not all the adsorbed 2,4-DCP was released into the solution, and only about $25 \%$ of it can be desorbed from the solid phase. The release of 2,4-DCP from the loaded nickel nanoparticles was very fast and its concentration reached maximum levels very quickly. For the nickel nanoparticles without acid-wash pretreatment, a relatively large fluctuation in the concentration of released 2,4-DCP was observed, which implies that multiple competitive interfacial reaction processes are involved. By comparison, for the nickel nanoparticles in the neutral system, the released 2,4-DCP concentration was stable over most of the desorption period. The trace amounts of 2,4DCP released into the solution indicates that complete degradation occurred under acidic conditions.

According to adsorption theory, both physical and chemical mechanisms may be involved in the sorption process of 2,4-DCP by nickel nanoparticles. The small fraction that was released into the solution when the loaded nickel nanoparticles were re-suspended in water for desorption (Figure 4) was probably due to the physical process. However, the majority of 2,4-DCP taken up by the nickel nanoparticles were not released easily, and most of them would combine with the nanoparticles by a chemical interaction. Previous studies using Fourier transform infrared analysis demonstrated that chlorophenols can be bound to 

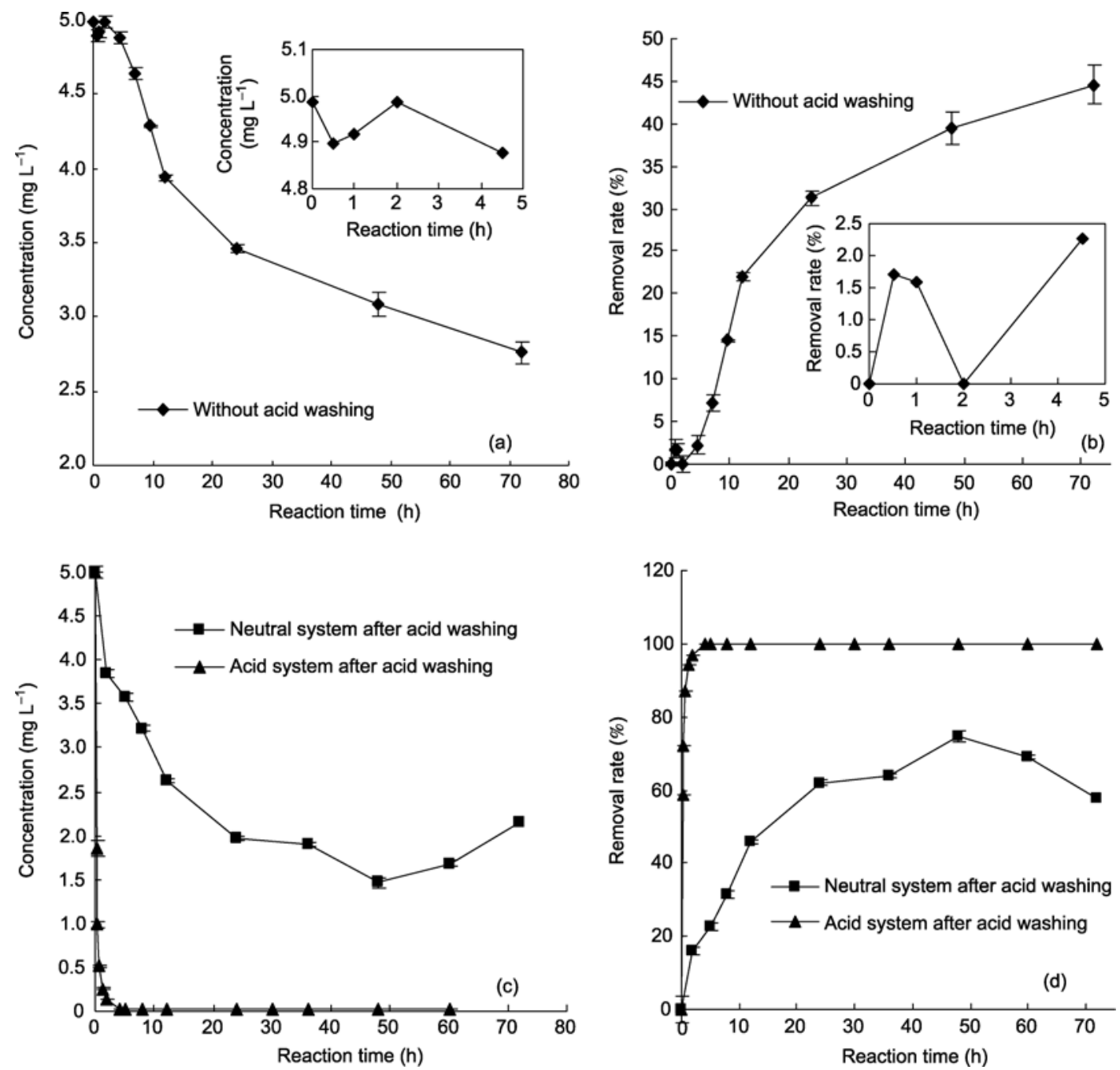

Figure 3 Residual concentration and removal rate of 2,4-DCP by nickel nanoparticles without acid-wash pretreatment (a) and (b) and after acid-wash pretreatment (c) and (d) of the nanoparticles.

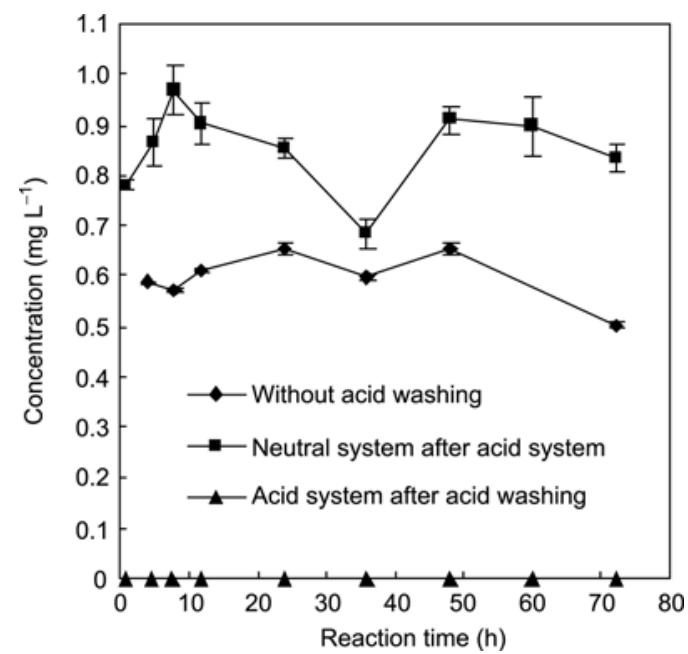

Figure 4 Desorption of 2,4-DCP from the loaded nickel nanoparticles in three different systems.

zero valent iron (ZVI) and its oxide surfaces through coordination of their functional groups, and is not removed by washing with water or through the degradation process. However, the presence of acid in the system largely facilitates the removal of chlorophenols by the dechlorination process.

In summary, during the uptake of 2,4-DCP by nickel nanoparticles in the three suspension systems, the removal of 2,4-DCP by untreated nickel nanoparticles was mainly by the adsorption process. By comparison, for nickel nanoparticles after acid-washing and under acidic conditions $(\mathrm{pH}$ $3-4)$, the dechlorination reaction was dominant. In general, nickel nanoparticles without acid-wash pretreatment usually have low reactivity in the dechlorination of 2,4-DCP, and the removal of 2,4-DCP was mainly by the adsorption of $\mathrm{NiO}$ passivation layers on their surfaces. Acid washing for activation removes the oxide layers and exposes large areas of fresh zero-valent nickel $\left(\mathrm{Ni}^{0}\right)$ surfaces to the solution. This greatly improves the 2,4-DCP degradation reactivity because of the strong reduction of $\mathrm{Ni}^{0}$. As the reaction continues, the solution $\mathrm{pH}$ increases and corrosion products of nickel hydroxides and/or oxides are formed on the particle 
surfaces, where the surface reactive sites are gradually occupied, leading to an incomplete dechlorination reaction. In the acidic system, the solution $\mathrm{pH}$ was adjusted by continuously adding $\mathrm{H}_{2} \mathrm{SO}_{4}$ to maintain the $\mathrm{pH}$ value of 3-4. In this way, accumulation of oxide layers on the particle surfaces is effectively blocked. The exposure of fresh surfaces and the high reactivity of nickel nanoparticles with a continuous supply of $\mathrm{H}^{+}$by $\mathrm{H}_{2} \mathrm{SO}_{4}$ both contribute to accelerating completion of the dechlorination reaction of 2,4-DCP.

The adsorption process is an important pathway for the uptake and removal of chlorophenol and chlorinated ethylene compounds in metal nanoparticle applications [16,17], and adsorption of chlorinated ethanes to graphitic inclusions in cast iron powders has been observed $[18,19]$. In the present study, nickel nanoparticles were produced via the carbonyl powder process, which introduced a small quantity of carbon to the nanoparticles (Table 1). Thus, the uptake and removal of 2,4-DCP by nickel nanoparticles in the neutral system may be because of a similar mechanism, in which adsorption by carbon components and nickel oxides are dominant.

\subsection{Adsorption and dechlorination of 2,4-DCP by nickel nanoparticles under acidic conditions at different temperatures}

As a solid-liquid two-phase reaction, there are three steps involved in the dechlorination of 2,4-DCP by nickel nanoparticles. First, the mass transfer of 2,4-DCP from solution to the metal surface of nickel. This is followed by adsorption and dechlorination of 2,4-DCP on the nickel surface. And finally, the dechlorination products desorb from the nickel surface and diffuse into the bulk solution. As all batch experiments in this investigation were carried out on a thermostatic orbital shaker with full agitation, the mass transfer control as in the first and third steps can be ruled out as governing the reaction rate. The adsorption process of 2,4-DCP on nanoscale nickel in the second step also proceeds easily. Therefore, dechlorination is likely the rate-determining step for the degradation of 2,4-DCP by nickel nanoparticles. The solution $\mathrm{pH}$ and temperature are two major factors known to influence the dechlorination reaction. It has been shown that the solution $\mathrm{pH}$ with nanoiron system changes slowly while that with nickel nanoparticles goes up rapidly, which implies that $\mathrm{H}^{+}$ions are extremely consumed in the latter. Consequently, we further investigated the adsorption and dechlorination of 2,4-DCP by nickel nanoparticles under acidic conditions.

Figure 5 shows the concentration and removal of 2,4DCP under acidic conditions at three different temperatures of $298 \mathrm{~K}, 306 \mathrm{~K}$, and $316 \mathrm{~K}$, respectively, along with the $\mathrm{H}_{2} \mathrm{SO}_{4}$ consumption and comparison with the blank control. The changes in the concentration and removal rate of 2,4-DCP with reaction time are illustrated in Figure 5(a) and (b). Under acidic conditions, $99 \%$ of 2,4-DCP was removed in less than $5 \mathrm{~h}$ at the room temperature. The removal and reaction rates increased rapidly as the temperature increased. Complete removal was obtained within $3 \mathrm{~h}$ at $306 \mathrm{~K}$. At $316 \mathrm{~K}$, $90 \%$ removal was achieved in only $30 \mathrm{~min}$ and complete removal within 2 h. Figure 5(c) compares 2,4-DCP concentrations in both the nanoscale nickel and blank control samples under acidic conditions. The acidic consumption at the three different temperatures is given in Figure 5(d), and the volume of $\mathrm{H}_{2} \mathrm{SO}_{4}$ required to maintain the $\mathrm{pH}$ at 3-4 increased with temperature and the 2,4-DCP removal. The introduction of $\mathrm{H}_{2} \mathrm{SO}_{4}$ obviously promoted degradation by optimizing the solution $\mathrm{pH}$ for dechlorination. Dissociated $\mathrm{H}^{+}$was also involved in the reaction. Dechlorination products such as 2-chlorophenol, 4-chlorophenol, and phenol were detected during the reaction, and phenol was the dominant product (Figure 6). The chemical reaction of 2,4-DCP on the nickel surface increased with temperature and this accelerated the entire dechlorination process. The dechlorination efficiency of 2,4-DCP under acidic conditions was very high with complete removal obtained after a few hours.

The kinetics of 2,4-DCP dechlorination by nickel nanoparticles under acidic conditions were evaluated using linear plots of $1 / C-1 / C_{0}$ versus reaction time at the three different temperatures (Figure 7). The second order rate law fit the data very well, and it can be expressed as follows: $-\mathrm{d} C / C^{2}=k \mathrm{~d} t$, or in the integrated form as: $1 / C=k t+1 / C_{0}$, where $C$ is the 2,4-DCP concentration in the reaction system, $C_{0}$ is its initial concentration, $t$ is reaction time, and $k$ is the rate constant, which can be obtained from the slope of graph. The rate constants for 2,4-DCP dechlorination at 298, 306, and $316 \mathrm{~K}$ were calculated at $0.02,0.2$, and $0.3(\mathrm{~g} \mathrm{~L} \mathrm{~h})^{-1}$, respectively. The parameters for linear regression equations are shown in Table 2 . In the neutral system, a pseudo-firstorder reaction was demonstrated with its rate constants of $0.0244,0.0379$ and $0.107 \mathrm{~h}^{-1}$ at the three different temperatures, respectively. This indicates that the addition of $\mathrm{H}_{2} \mathrm{SO}_{4}$ promotes 2,4-DCP dechlorination to become a second order reaction, which agrees with the results as given in Figure 5.

A previous study [19] suggested that dechlorination of 2,4-DCP by nickel nanoparticles proceeds mainly via the following three pathways: (1) the nickel nanoparticles react with $\mathrm{H}^{+}$to produce atomic $\mathrm{H}$; (2) the atomic $\mathrm{H}$ reduces 2,4DCP to the degradation products; and (3) as the reaction proceeds and the acid is consumed, nickel ions react with $\mathrm{OH}^{-}$and form a sediment coated on the particles, which leads to formation and accumulation of hydroxide/oxide layers on the particle surfaces and this stops nickel nanoparticles from reacting further. At the same time, the coated oxide layers may adsorb the reactants to some extent, and in this case, reduction will not occur, which results in incomplete degradation. Dechlorination of 2,4-DCP occurred mainly by reductive addition of atomic hydrogen under catalysis by zero-valent metal nanoparticles. However, $\mathrm{H}_{2}$ by itself is an 

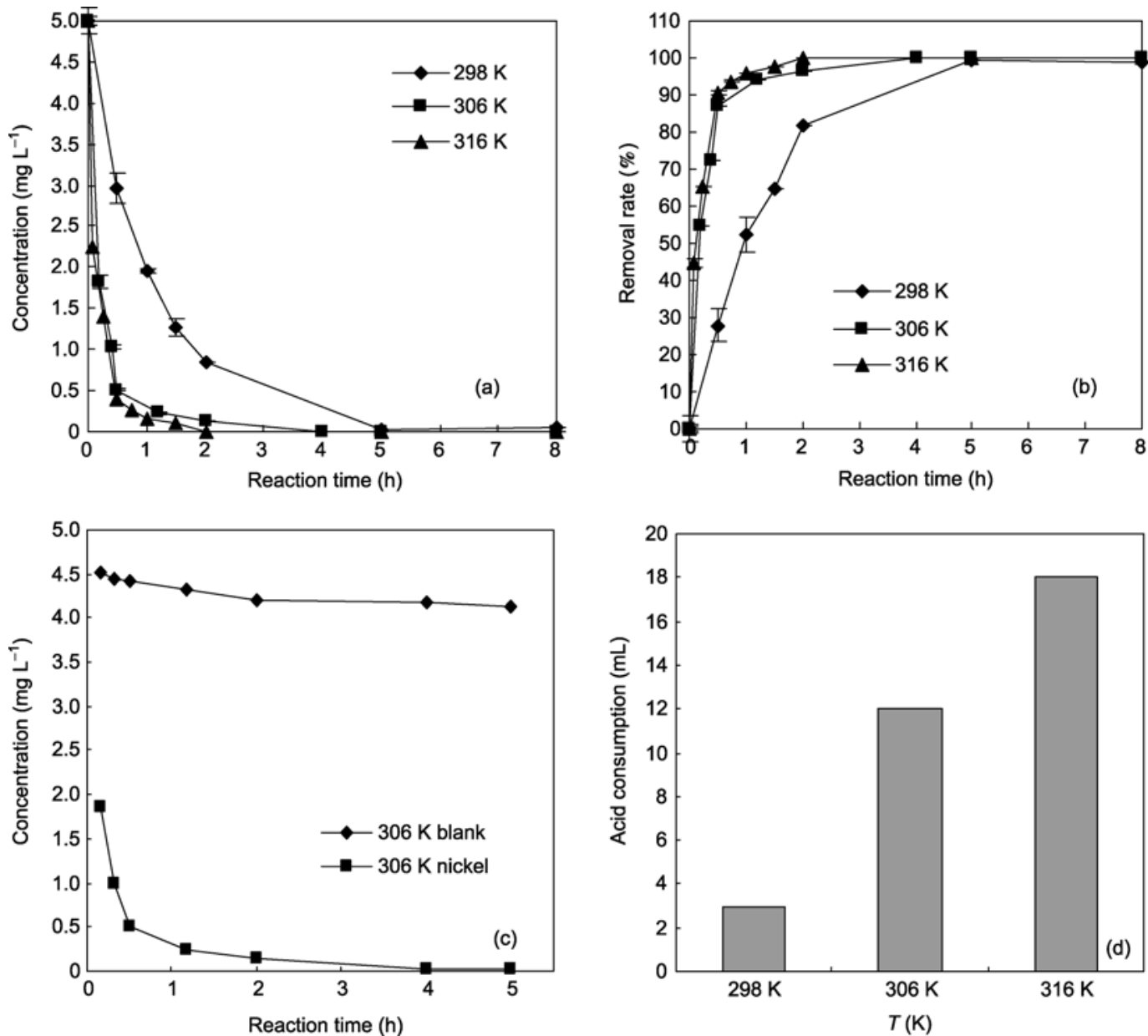

Figure 5 The concentration (a) and removal (b) of 2,4-DCP by nickel nanoparticle suspensions under acidic conditions at three different temperatures of $298 \mathrm{~K}, 306 \mathrm{~K}$, and $316 \mathrm{~K}$, respectively, along with comparison of the 2,4-DCP residual concentration (c) for the nickel nanoparticle suspension and control blank and the volume of acid $\left(1: 200\right.$ in $v / v$ ratio of $\mathrm{H}_{2} \mathrm{SO}_{4}$ : water) consumption (d).

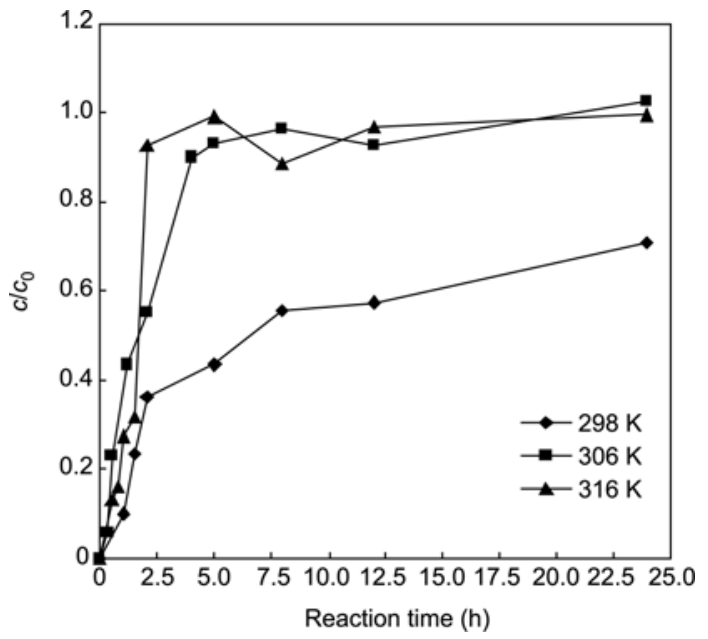

Figure 6 Formation of the dechlorination product as phenol at 298, 306, and $316 \mathrm{~K}$, respectively.

inactive reducing agent, and reduction is only possible when a catalyst or substrate is available in the reaction system. As a widely used hydrogenation catalyst, nickel has a good

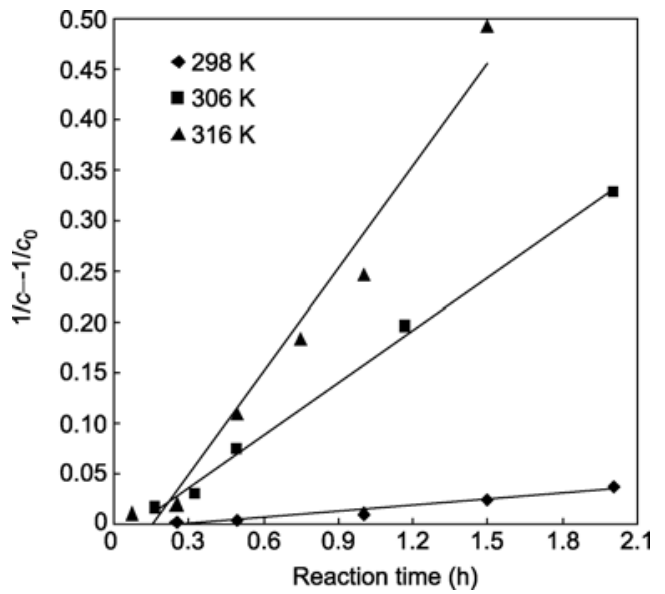

Figure 7 Linear plots fitted by the second-order kinetic model for 2,4-DCP dechlorination by nickel nanoparticles under acidic conditions at 298,306 , and $316 \mathrm{~K}$, respectively.

capacity for adsorptive storage of $\mathrm{H}_{2}$ and strong ability to break molecular $\mathrm{H}_{2}$ into atomic $\mathrm{H}$. Consequently, it can largely accelerate the dechlorination of 2,4-DCP. However, 
Table 2 Linear regression equation parameters for 2,4-DCP degradation by nickel nanoparticles

\begin{tabular}{ccc}
\hline$T(\mathrm{~K})$ & Fitted equation & $R^{2}$ \\
\hline 298 & $Y=0.02 x-5 \times 10^{-6}$ & 0.9653 \\
306 & $Y=0.2 x-2 \times 10^{-5}$ & 0.9965 \\
316 & $Y=0.3 x-5 \times 10^{-5}$ & 0.9714 \\
\hline
\end{tabular}

in the neutral system, the interaction of nickel nanoparticles with water to generate atomic $\mathrm{H}$ is extremely slow, and this results in a low efficiency of dechlorination. While in the acidic system, a large quantity of hydrogen is present from the acid and it is essential to the dechlorination reaction, which will facilitate its adsorptive enrichment and the catalytic function of nickel nanoparticles. The dechlorination efficiency is therefore greatly improved. Besides as a proton source in the dechlorination, the $\mathrm{H}_{2} \mathrm{SO}_{4}$ may also act as follows: (1) by eroding nickel to expose the fresh surfaces of nanoparticles, which will accelerate dechlorination; (2) by preventing formation and accumulation of insoluble oxide/ hydroxide layers on the particle surfaces; and (3) by preventing adsorption of 2,4-DCP target reactants to the loosely bound oxide layers on the surface of nanoparticles, which will promote a complete degradation.

\subsection{Adsorption and desorption of phenol as dechlori- nation product by nickel nanoparticles under neutral conditions}

Degradation of 2,4-DCP by nickel nanoparticles usually remains at the dechlorination stage, and the ring-opening for a further degradation will not occur. Therefore, phenol turns out to be as the major degradation product. However, large fluctuations in phenol concentration were observed when 2,4-DCP was dechlorinated in the neutral system, which can be attributed to the adsorption of oxide layers on the nanoparticles. A further study was then performed to evalu- ate the uptake and desorption behaviors of phenol by nickel nanoparticles. In this case, the initial phenol concentration of $2.8 \mathrm{mg} / \mathrm{L}$ was used, and the total reaction volume was $100 \mathrm{~mL}$.

As can be seen from Figure 8, the uptake of phenol by nickel nanoparticles in the neutral system was $20 \%-35 \%$ in relation to $0.28 \mathrm{mg}$ of initial phenol. Subsequently, $20 \%$ of it was further released into the solution during the desorption process. In this context, the adsorption capacity of phenol by nickel nanoparticles was less than $0.1 \mathrm{mg}$. According to the stoichiometric calculation with complete dechlorination of 2,4-DCP, the maximum phenol quantity released by the dechlorination process was $0.56 \mathrm{mg}$ in relation to $1 \mathrm{mg}$ of initial 2,4-DCP quantity. Because the 2,4DCP removal by nickel nanoparticles in the neutral system was not very high $(<70 \%$, Figure 3$)$, and quite a large fraction of it will be immobilized via adsorption process. These adsorption and desorption processes, competitive together with the dechlorination reaction, were involved in the neutral system, and lead to fluctuations and irregular changes in the phenol concentration, which was in agreement with our experimental results in this section.

\section{Conclusions}

Nickel nanoparticles manufactured by carbonyl powder technology have an average size of $10-20 \mathrm{~nm}$ in diameter with a specific surface area of $32.40 \mathrm{~m}^{2} / \mathrm{g}$. Compared with iron nanoparticles of similar manufacturing process, oxide passivation layers present on nickel are very thin. These oxide layers and carbonic inclusions are dominant pathways for 2,4-DCP adsorption.

Acid washing favored the removal of oxide layers and increased the 2,4-DCP dechlorination efficiency by nickel nanoparticles. Under neutral conditions, however, dechlorination was blocked and incomplete degradation was obvious
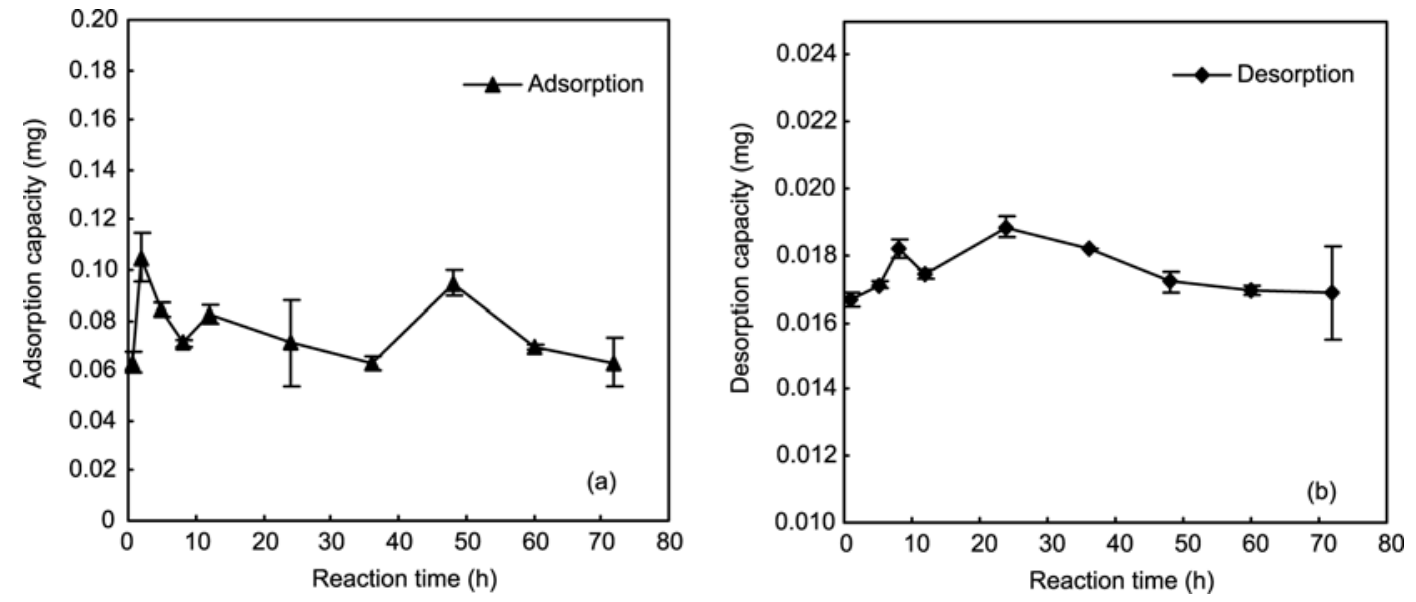

Figure 8 Adsorption (a) and desorption (b) of phenol as dechlorination product of 2,4-DCP by nickel nanoparticles under neutral conditions. 
due to formation and accumulation of hydroxide/oxide layers on particle surfaces as the solution $\mathrm{pH}$ was increased in the reaction process. The introduction of $\mathrm{H}_{2} \mathrm{SO}_{4}$ can promote the degradation process by providing appropriate $\mathrm{pH}$ conditions and a fresh supply of $\mathrm{H}^{+}$ions in the dechlorination reaction.

Dechlorination of 2,4-DCP by nickel nanoparticles was greatly improved under acidic conditions ( $\mathrm{pH} 3-4)$, and almost complete removal of 2,4-DCP was reached within $4 \mathrm{~h}$. Phenol was detected as dominant product in the dechlorination. The degradation reaction was found to follow the second order kinetic model, and the rate constants at three different temperatures of 298,306 , and $316 \mathrm{~K}$ were 0.02 , 0.2 and $0.3(\mathrm{gL} \mathrm{h})^{-1}$, respectively.

This work was supported by the National Basic Research Program of China (2007CB407304) and the National Science \& Technology Major Project of China (2009ZX07209-004).

1 Zhang W X, Wang C B, Lien H L. Treatment of chlorinated organic contaminants with nanoscale bimetallic particles. Catal Today, 1998, 40: 387-395

2 Ball P, Garwin L. Science at the atomic scale. Nature, 1992, 355: 761-766

3 Choe S, Chang Y Y, Hwang K Y, et al. Kinetics of reductive denitrification by nanoscale zero-valent iron. Chemosphere, 2000, 41: 1307-1311

4 Masciangioli T, Zhang W X. Environmental technologies at the nanoscale. Environ Sci Technol, 2003, 37: 102A-108A

5 Zhang W X. Nanoscale iron particles for environmental remediation: An overview. J Nanopart Res, 2003, 5: 323-332

6 Song H, Carraway E. Reduction of chlorinated ethanes by nanosized zero-valent iron: Kinetics, pathways and effects of reaction conditions. Environ Sci Technol, 2005, 39: 6237-6245
7 Alessi D S, Zhao H L. Synergistic effect of cationic surfactants on perchloroethylene degradation by zero-valent iron. Environ Sci Technol, 2001, 35: 3713-3717

8 Lowry G V, Johnson K M. Congener specific dechlorination of dissolved PCBs by microscale and nanoscale zero valent iron in a water/ methanol solution. Environ Sci Technol, 2004, 38: 5208-5216

9 Jovanovic G N, Znidarsic P P, Sakrittichai P, et al. Dechlorination of p-chlorophenol in a microreactor with bimetallic $\mathrm{Pd} / \mathrm{Fe}$ catalyst. Ind Eng Chem Res, 2005, 44: 5099-5106

10 Dombek T, Davis D, Stine J, et al. Degradation of terbutylazine (2chloro-4-ethylamino-6-terbutylamino-1,3,5-triazine), deisopropyl atrazine (2-amino-4-chloro-6-ethylamino-1,3,5-triazine), and chlorinated dimethoxy triazine (2-chloro-4,6-dimethoxy-1,3,5-triazine) by zero valent iron and electrochemical reduction. Environ Poll, 2004, 129: 267-275

11 Keum Y S, Li Q X. Reductive debromination of polybrominated diphenyl ethers by zero-valent iron. Environ Sci Technol, 2005, 39: 2282-2286

12 Hou C F, Ge X P, Zhou Y M, et al. Characterization of nanoscale iron and its degradation of 2,4-dichlorophenol. Chinese Sci Bull, 2010, 55: 350-357

13 Kim Y H, Carraway E R. Dechlorination of pentachlorophenol by zero valent iron and modified zero valent irons. Environ Sci Technol, 2000, 34: 2014-2017

14 Rong M Z, Zhang M Q, Wang H B, et al. Surface modification o magnetic metal nanoparticles and its influence on the performance of polymer composites. J Poly Sci (Part B): Polymer Phys, 2003, 41: 1070-1084

15 Kung K S, McBride M B. Bonding of chlorophenol on iron and aluminum-oxides. Environ Sci Technol, 1991, 25: 702-709

16 Burris D R, Campbell T J, Manoranjan V S. Sorption of trichloroethylene and tetrachloroethylene in a batch reactive metallic ironwater system. Environ Sci Technol, 1995, 29: 2850-2855

17 Allen-King R M, Halket R M, Burris D R. Reductive transformation and sorption of cis- and trans- 1,2-dichlorothene in a metallic iron/water system. Environ Toxicol Chem, 1997, 16: 424-429

18 Deng B, Campbell T J, Burris D R. Reduction of vinyl chloride in metallic iron-water systems. Environ Sci Technol, 1997, 31: 1185-1190

19 Liu Y H, Yang F L, Yue P L, et al. Catalytic dechlorination of chlorophenols in water by palladium/iron. Water Res, 2001, 35: $1887-1890$

Open Access This article is distributed under the terms of the Creative Commons Attribution License which permits any use, distribution, and reproduction in any medium, provided the original author(s) and source are credited. 